\title{
Lobectomy with pulmonary artery angioplasty for lung cancer using video-assisted thoracic surgery versus open thoracotomy: a retrospective propensity matched analysis
}

\author{
Chengwu Liu ${ }^{1,2 \#}$, Zhenyu Yang ${ }^{1,2 \#}$, Chenglin Guo ${ }^{1,2}$, Yunke Zhu ${ }^{1,2}$, Qiang Pu ${ }^{1,2}$, Jiandong Mei ${ }^{1,2}$, Lin $^{M^{1,2}}{ }^{\text {, }}$ \\ Feng $\operatorname{Lin}^{1,2}$, Lunxu Liu ${ }^{1,2}$ \\ ${ }^{1}$ Department of Thoracic Surgery, West China Hospital, Chengdu, China; ${ }^{2}$ Western China Collaborative Innovation Center for Early Diagnosis and \\ Multidisciplinary Therapy of Lung Cancer, Sichuan University, Chengdu, China \\ Contributions: (I) Conception and design: C Liu, Z Yang, L Liu; (II) Administrative support: L Liu; (III) Provision of study materials or patients: C \\ Liu, Q Pu, J Mei, L Ma, Y Zhu, F Lin, L Liu; (IV) Collection and assembly of data: C Liu, Z Yang, C Guo; (V) Data analysis and interpretation: C \\ Liu, Z Yang, C Guo; (VI) Manuscript writing: All authors; (VII) Final approval of manuscript: All authors. \\ \#These authors contributed equally to this work. \\ Correspondence to: Lunxu Liu, MD, PhD. Department of Thoracic Surgery, West China Hospital, Sichuan University, No. 37, Guoxue Alley, \\ Chengdu 610041, China. Email: lunxu_liu@aliyun.com.
}

\begin{abstract}
Background: Pulmonary artery angioplasty (PAA) is an important surgical procedure to complete radical resection with maximum preservation of functioning pulmonary tissues when the pulmonary artery is involved. In this study, we aimed to evaluate the short- and long-term outcomes of PAA using video-assisted thoracic surgery (VATS) versus open thoracotomy (OT) in lung cancer surgery.

Methods: We retrospectively reviewed 214 patients who underwent PAA between November 2005 and October 2016. Propensity score matching (PSM) was applied to reduce confounding effects. The survival outcomes were assessed using Kaplan-Meier estimates and Cox regression analysis.

Results: The final sample included 203 patients (28 patients in the VATS group and 175 patients in the OT group). There were no differences between the two groups in operative time, blood loss, duration of chest tube drainage, postoperative hospital stays, margin status, postoperative morbidity and mortality, and number of N1 and N2 stations or number of N1 and N2 lymph nodes both before and after matching. At a median follow-up period of 43 (range, 6 to 158) months, the 5-year overall survival (OS) and recurrence-free survival (RFS) of the overall cohort were $47.9 \%$ and $42.1 \%$, respectively. The 5-year OS and RFS were comparable between the VATS and OT groups both in the overall cohort and the matched cohort. The VATS procedure was found not to have a prognostic impact on either OS (hazard ratio, 1.17; 95\% CI: 0.60 to 2.30, P=0.647) or RFS (hazard ratio, 1.14 ; $95 \%$ CI: 0.62 to $2.10, \mathrm{P}=0.666$ ).
\end{abstract}

Conclusions: VATS PAA is associated with comparable short- and long-term outcomes in selected patients with lung cancer compared with OT.

Keywords: Lung cancer; video-assisted thoracic surgery (VATS); thoracotomy; pulmonary artery; angioplasty

Submitted Jul 22, 2021. Accepted for publication Aug 26, 2021.

doi: $10.21037 /$ tlcr-21-607

View this article at: https://dx.doi.org/10.21037/tlcr-21-607

\section{Introduction}

Parenchyma-preserving procedures contribute to preservation of pulmonary function and quality of life in the patients with and without compromised cardiopulmonary function (1,2). A lobectomy with partial removal and reconstruction of the pulmonary artery $(\mathrm{PA})$ allows the preservation of patients' functioning pulmonary tissues. The surgical outcomes of PA angioplasty (PAA) were 
found to be not only equal to those of a bronchial sleeve lobectomy but even better than those of pneumonectomy, if adequate resection is accomplished (3-8). Therefore, radical resection of the tumor with as much parenchyma preserved as possible is demanding and crucial for lung cancer surgery. Every effort should be made to avoid pneumonectomy as much as possible. Tumors or satellite lymph nodes can involve the PA to various degrees, from partial infiltration to a more extensive and even circumferential invasion. This heterogeneous presentation may require either partial removal of the arterial wall or even segmental resection of the PA followed by reconstruction $(9,10)$. Pulmonary artery angioplasty (PAA) on its own is a technically challenging procedure. Sometimes, bronchoplasty is also indicated for the same patient. Therefore, lobectomy with PAA with or without bronchoplasty even if performed using conventional open thoracotomy (OT) is not popular among thoracic surgeons.

Although video-assisted thoracic surgery (VATS) lobectomy is regarded as a recommended procedure for resectable lung cancer and has been adopted for complex procedures such as completion pneumonectomy (11), bronchial sleeve lobectomy (12), and similar procedures, it seems to be extremely difficult to perform angioplasty using VATS procedures due to various technical limitations, including prevention of catastrophic bleeding, controlling the mainstem of the PA, and suturing or anastomosis under thoracoscopy. Nakanishi et al. (13) reported the initial experience of VATS lobectomy with partial removal of the PA. Subsequently sporadic studies reported the feasibility and safety of the VATS procedure in performing PAA (14-19). However, these reports were limited in that they were single case reports or small case series, and that they did not address the long-term outcomes of the VATS procedure for PAA. Here, we report the first study that compares the short- and long-term outcomes of lobectomy with PAA using VATS with those of OT. We present the following article in accordance with the STROBE reporting checklist (available at https://dx.doi.org/10.21037/tlcr-21-607) (20).

\section{Methods}

This retrospective study was conducted using data collected from a prospectively maintained database, the Western China Lung Cancer (WCLC) database, which included all the lung cancer patients who underwent surgery at the Department of Thoracic Surgery, West China Hospital, since September 2005. The protocol of this study was approved by the institutional review board of West China Hospital (No. 2020-939). All procedures performed in this study involving human participants were in accordance with the Declaration of Helsinki (as revised in 2013).

\section{Patients}

Between November 2005 and October 2016, patients with lung cancer underwent lobectomy with resection and reconstruction of the PA were included. Patients with unanticipated metastases or missing value for follow-up and clinicopathological data were excluded from the final analysis. Patients were grouped into the VATS group or OT group according to the surgical approach used (Figure 1). Written informed consent was obtained from each patient before surgery. The pathologic parameters were reviewed again, and the patients were restaged according to the eight edition of the lung cancer staging system proposed by the International Association for the Study of Lung Cancer (IASLC).

\section{Operative procedure}

Lobectomy and systemic lymph node dissection were performed either through a thoracotomy or VATS approach under general anesthesia with single lung ventilation. The extent of PA invasion was carefully assessed before choosing an appropriate approach for resection and reconstruction. Typically, the pulmonary vein and arterial branches that were not involved in the tumor were managed first. The order of management of the fissures, arterial branches, and the bronchus was not identical. For those cases where sleeve resection of the bronchus and/or PA was indicated, dissection of the peri-hilar and mediastinal lymph nodes was performed in advance to increase the space available for resection and reconstruction of the bronchus and/or PA.

Resection and reconstruction of the PA were performed using different methods according to the specific circumferences of tumor invasion. First, for patients with less than $30 \%$ of the circumference of the PA invaded, two methods were adopted to accomplish partial removal of the side wall and reconstruction of the PA. One method was to perform angioplasty directly using a mechanical suture technique as described in our former study (21) and Xu's report (22). This method was performed only for cases with a small portion of the PA invaded (e.g., no more than a quarter of the circumference). It could be performed with the proximal trunk of the PA either clamped or not. 


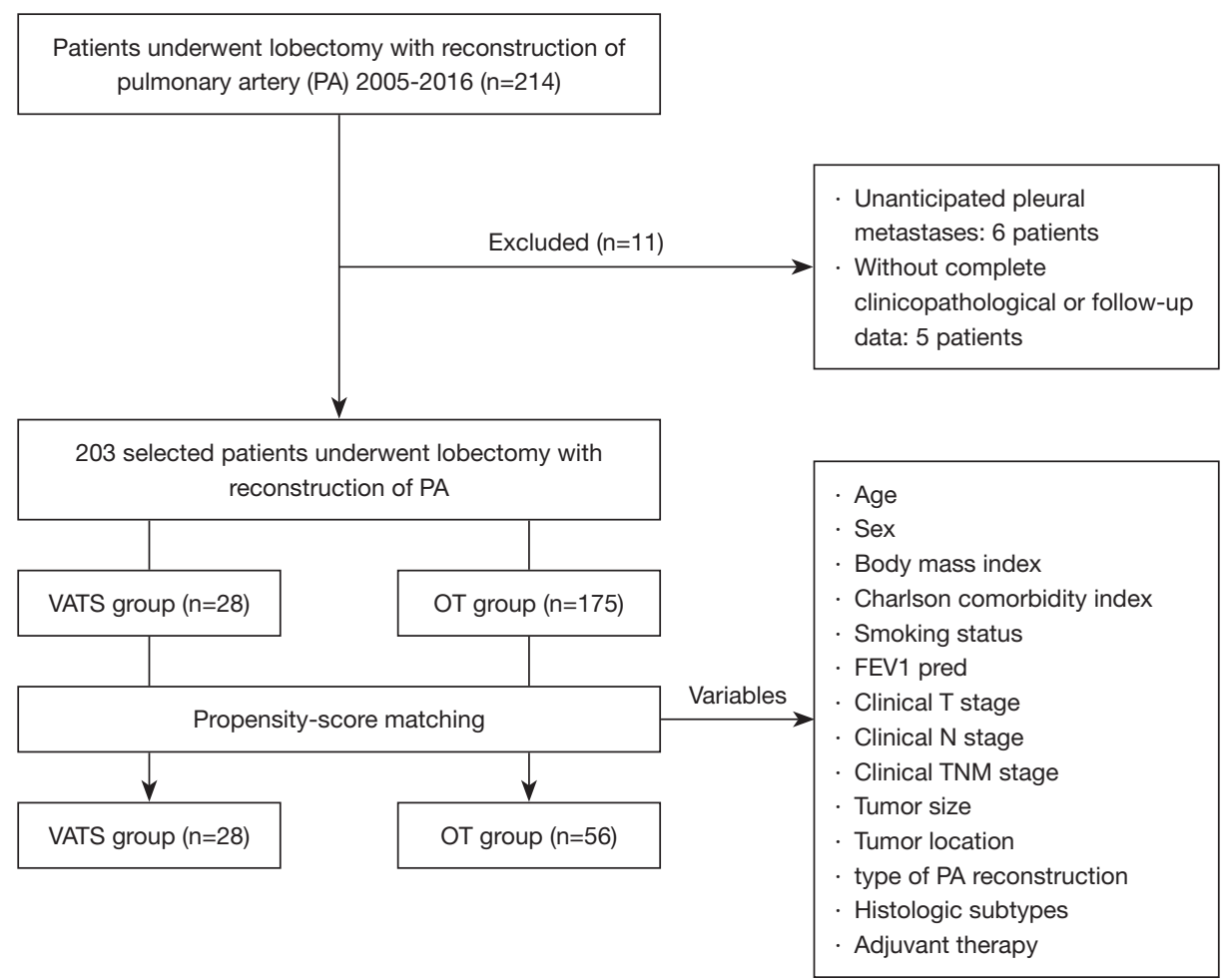

Figure 1 Flow chart diagram of patient selection. PA, pulmonary artery; VATS, video-assisted thoracic surgery; OT, open thoracotomy; FEV1, forced expiratory volume in 1 second.

The other method was to cut the side wall involved and perform manual suture with both the proximal trunk of the PA and the interlobar PA clamped with two Satinsky clamps or atraumatic bulldog clamps during a thoracotomy or with detachable atraumatic endoscopic bulldog clamps (Aesculap, Inc., Center Valley, PA, USA) during a VATS procedure (Figure 2). Intravenous injection of heparin was not required. However, diluted heparin $(25 \mathrm{U} / \mathrm{mL})$ was used to wash the endangium of the PA before suturing. Then the PA was repaired via a running suture using 4-0 Prolene (Ethicon Inc., Cornelia, GA, USA) stiches. Before tying the knot, the distal clamps were removed to vent gas. Finally, the proximal clamps were removed slowly. For patients with more extended invasion, we commonly chose to perform a circumferential resection of the artery and end-to-end anastomosis (sleeve reconstruction) rather than a patchy reconstruction or interposition of a prosthetic conduit. The main PA and interlobar artery were clamped first. After removal of the specimen, the reconstruction was performed via continuous suture with 5-0 Prolene stitches. In some cases, bronchoplasty was indicated and performed simultaneously. In that case, the anastomosis of the bronchus was performed followed by anastomosis of the PA (Figure 3). The detailed techniques of reconstruction using VATS were also described in our previous publication (19).

\section{Follow up}

All patients were followed up postoperatively following a set schedule, which included physical examinations, laboratory tests, chest and upper abdominal computed tomography (CT), brain magnetic resonance imaging (MRI) or CT every 3-4 months for the first 2 years, every 6 months for the next 3 years, and then yearly thereafter. Wholebody bone scintigraphy was performed once a year, and a positron emission tomography-CT scan was also conducted if necessary. The follow-up data were obtained from medical records or collected from patients or their relatives by telephone interview. If the patient was lost to follow up, the survival information was obtained from the National Death Registration System as an alternative. The followup data were updated until September 2019. Perioperative 30-day mortality was defined as any death within the first 30 days after surgery or hospitalization. Postoperative 

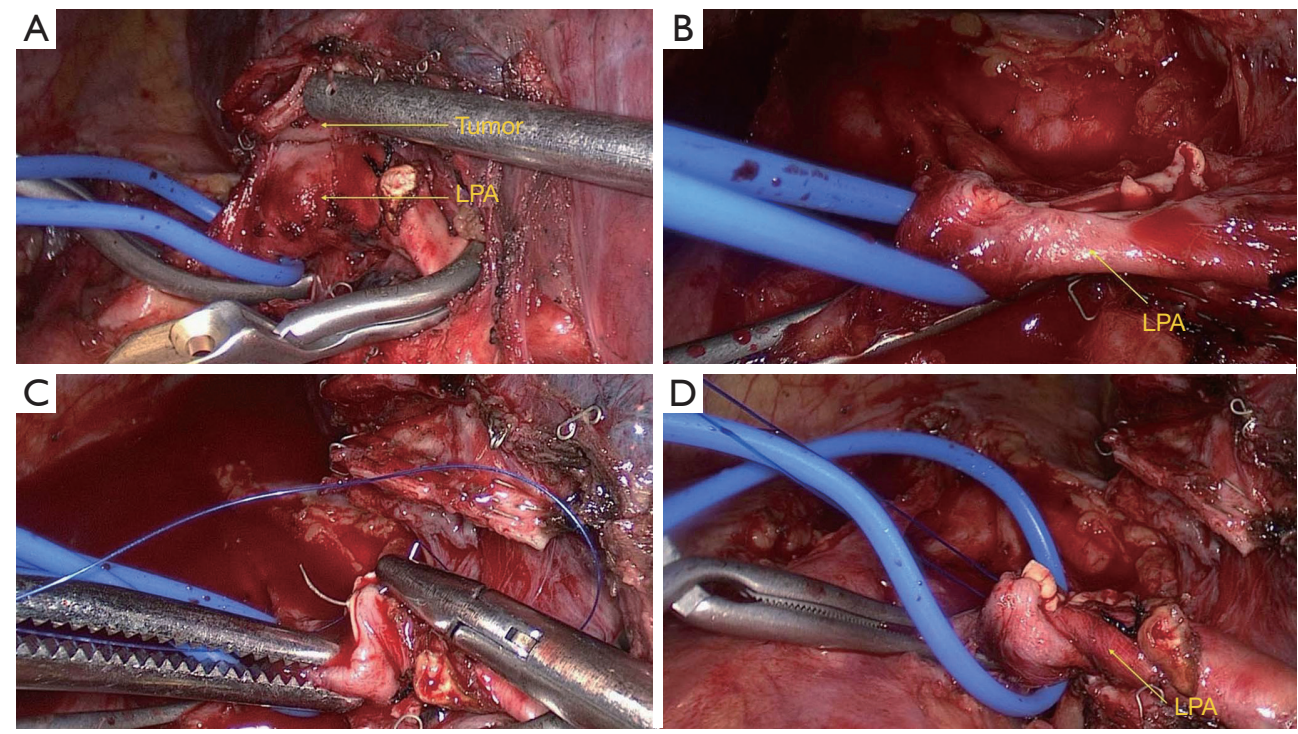

Figure 2 Partial removal of the side wall and reconstruction of the PA. (A) Dissect and clamp the left PA trunk and interlobar PA using detachable atraumatic endoscopic bulldog clamps; (B) cut the invaded side wall of the PA; (C) perform manual suture using 4-0 Prolene stiches; (D) Tie the knot and complete PA reconstruction. PA, pulmonary artery; LPA, left pulmonary artery.
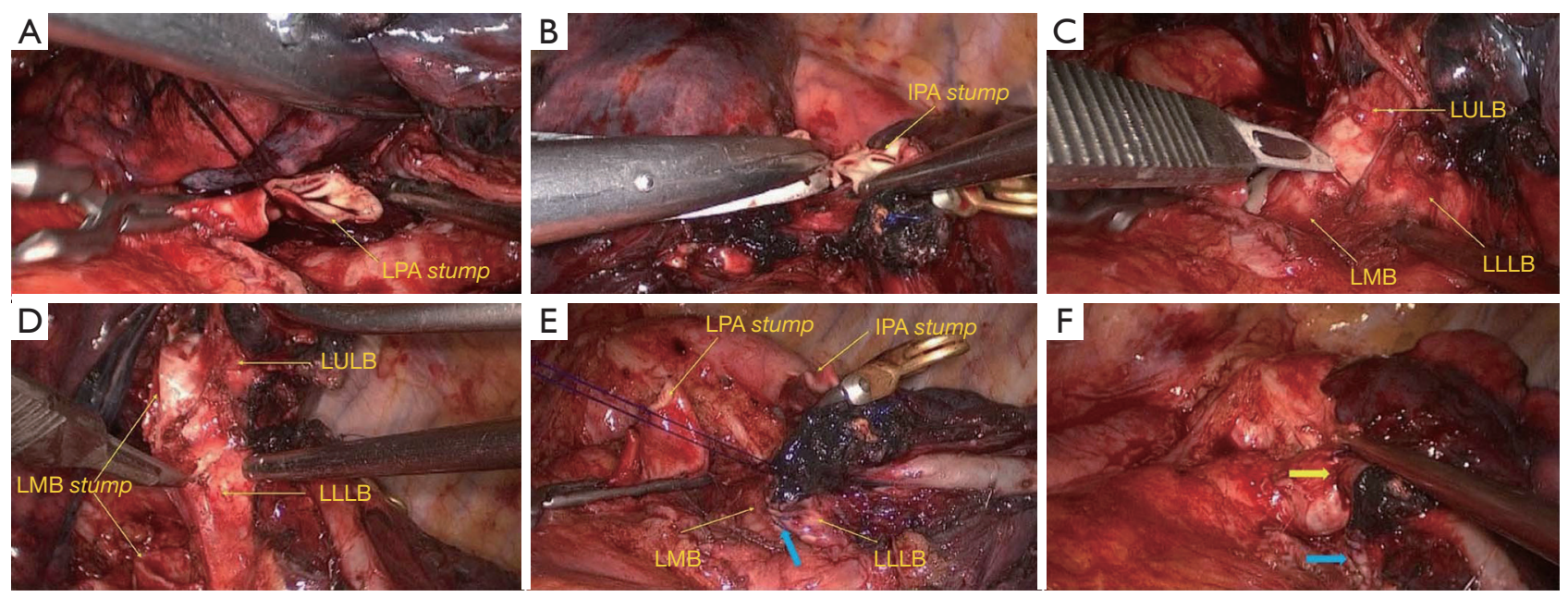

Figure 3 Double sleeve reconstruction of the PA and bronchus. (A,B) Segmental resection of the left PA; (C,D) cut the left main bronchus and lower lobe bronchus; (E,F) perform end-to-end anastomosis of the bronchus and PA using 3-0 and 5-0 Prolene stiches, respectively. PA, pulmonary artery; LPA, left pulmonary artery; LMB, left main bronchus; LULB, left upper lobe bronchus; LLLB, left lower lobe bronchus; blue arrow, anastomosis of the bronchus; yellow arrow, anastomosis of the PA.

90-day mortality was also recorded and defined as any death within 90 days after surgery. Overall survival (OS) was defined as the time from the date of surgery until death from any cause or the last follow up. Recurrence-free survival (RFS) was calculated as the time interval from the date of surgery until any local or distant recurrence, or the last follow up. Both OS and RFS were calculated in months.

\section{Statistical analyses}

Student's $t$-tests and Mann-Whitney $\mathrm{U}$ tests were used to compare the distributions of continuous data between the two 
groups wherever applicable. A Pearson $\chi^{2}$ test was employed to compare the frequencies of categorical measures, and a Fisher's exact test was used if there were expected counts $<10$. To minimize selection bias between the two groups, propensity score matching (PSM) was performed with a ratio of $1: 2$ of the number of patients in the VATS group versus those in the OT group, using a multiple logistic regression model. The following covariates associated with survival were included: age; sex; body mass index (BMI); smoking history; forced expiratory volume in 1 second percent predicted (FEV1 pred); comorbidity; clinical tumor (T) stage; clinical node $(\mathrm{N})$ stage; clinical tumor, node, and metastasis (TNM) stage; tumor size; tumor location; type of PA reconstruction; histological subtype; and adjuvant therapy. Finally, 28 patients in the VATS group and 56 patients in the OT group were matched. The patient inclusion flow chart is shown in Figure 1. The Kaplan-Meier method and the log-rank test were used to analyze the OS and RFS of the two groups before and after matching. Univariable Cox proportionalhazards regression analyses for OS and RFS were performed in the propensity-matched cohort categories, and those with $\mathrm{P}<0.1$ were subsequently included in multivariable analyses. Categorical data were reported as counts and percentages, and continuous variables were reported as means \pm standard deviations. The level of statistical significance was set at $\mathrm{P}<0.05$. All comparisons were two-tailed. Statistical tests were performed using R Version 3.5.1 (R Foundation for Statistical Computing, Vienna, Austria).

\section{Results}

\section{Clinical characteristics}

In total, 214 patients underwent lobectomy with PAA were included. Six patients with unanticipated pleural metastases, four patients without follow-up data, and one patient with incomplete clinicopathological data were excluded from the final analysis. As a result, 203 patients were included in the final sample of this study (28 patients in the VATS group and 175 patients in the OT group) (Figure 1). There were 50 patients with partial removal and reconstruction of the PA (13 using VATS and 37 using OT), 46 patients with sleeve reconstruction of the PA (5 using VATS and 41 using OT), 29 patients with partial removal and reconstruction of the PA plus bronchoplasty (3 using VATS and 26 using OT), and 78 patients with sleeve reconstruction of the PA and bronchoplasty (6 using VATS and 71 using OT). The detailed clinical characteristics of the two groups both before and after matching are shown in Table 1. Before matching, there were more patients with compromised pulmonary function (FEV1 pred $<80 \%)(\mathrm{P}=0.014)$ and tumors $>5 \mathrm{~cm}$ in the $\mathrm{OT}$ groups $(\mathrm{P}=0.007)$. In addition, the smoking status $(\mathrm{P}=0.063)$, type of $\mathrm{PA}$ reconstruction $(\mathrm{P}=0.057)$, and histological subtypes $(\mathrm{P}=0.062)$ were marginally different between the two groups. After matching there were no significant differences between the two groups in age, gender, sex, BMI, smoking status, FEV1 pred, Charlson comorbidity index, clinical T stage, clinical N stage, clinical TNM stage, tumor size, tumor location, type of PA reconstruction, histologic subtype, pathological TNM stage, and adjuvant therapy.

\section{Perioperative outcomes}

The perioperative outcomes of the two groups both before and after matching are shown in Table 2. Seven patients underwent conversion from VATS to OT with a conversion rate of $25 \%(7 / 28)$. The reasons of conversion included severe infiltration of the tumor around the lobar hilum (5 patients), invasion of the chest wall (1 patient), and invasion of the superior vena cava (1 patient). When analyzing the perioperative outcomes, the data of these 7 patients were also included in the VATS group. In the matched cohort, although not significantly different, the VATS procedure was associated with longer operative time $(217.86 \pm 67.69 v s$. $202.93 \pm 65.90 \mathrm{~min}, \mathrm{P}=0.273$ ) and less intraoperative blood loss (157.50 \pm 72.91 vs. $188.57 \pm 125.57 \mathrm{~mL}, \mathrm{P}=0.399)$ compared with the OT. There were no differences between the two groups in duration of chest tube drainage, postoperative hospital stays, margin status, and number of dissected N1 and $\mathrm{N} 2$ stations or number of N1 and N2 lymph nodes both before and after matching. The rate of major postoperative complications of the whole cohort was 35.96\% (73/203), including 51 cases of prolonged drainage ( $>7$ days), 31 cases of pneumonia, 17 cases of prolonged air leakage ( $>5$ days), 4 cases of bronchopleural fistula, 4 cases of respiratory failure, 3 cases of chylothorax, 3 cases of wound infection, 2 cases of pulmonary embolism, 2 cases of reoperation, 2 cases of arrhythmia, 1 case of urinary infection, and 1 case of cardiac failure. Among these patients, 36 patients had more than one kind of postoperative complication. The 30- and 90-day mortality of the whole cohort were $1.5 \%(3 / 203)$ and $4.9 \%$ (10/203), respectively. All the three patients who died within 30 days were in the OT group. Two patients died from septic shock, and respiratory failure resulted from bronchopleural fistula 13 and 29 days after the operation, respectively. One patient died from massive bleeding 9 days after the operation. 
Table 1 Patients' characteristics before and after propensity score matching

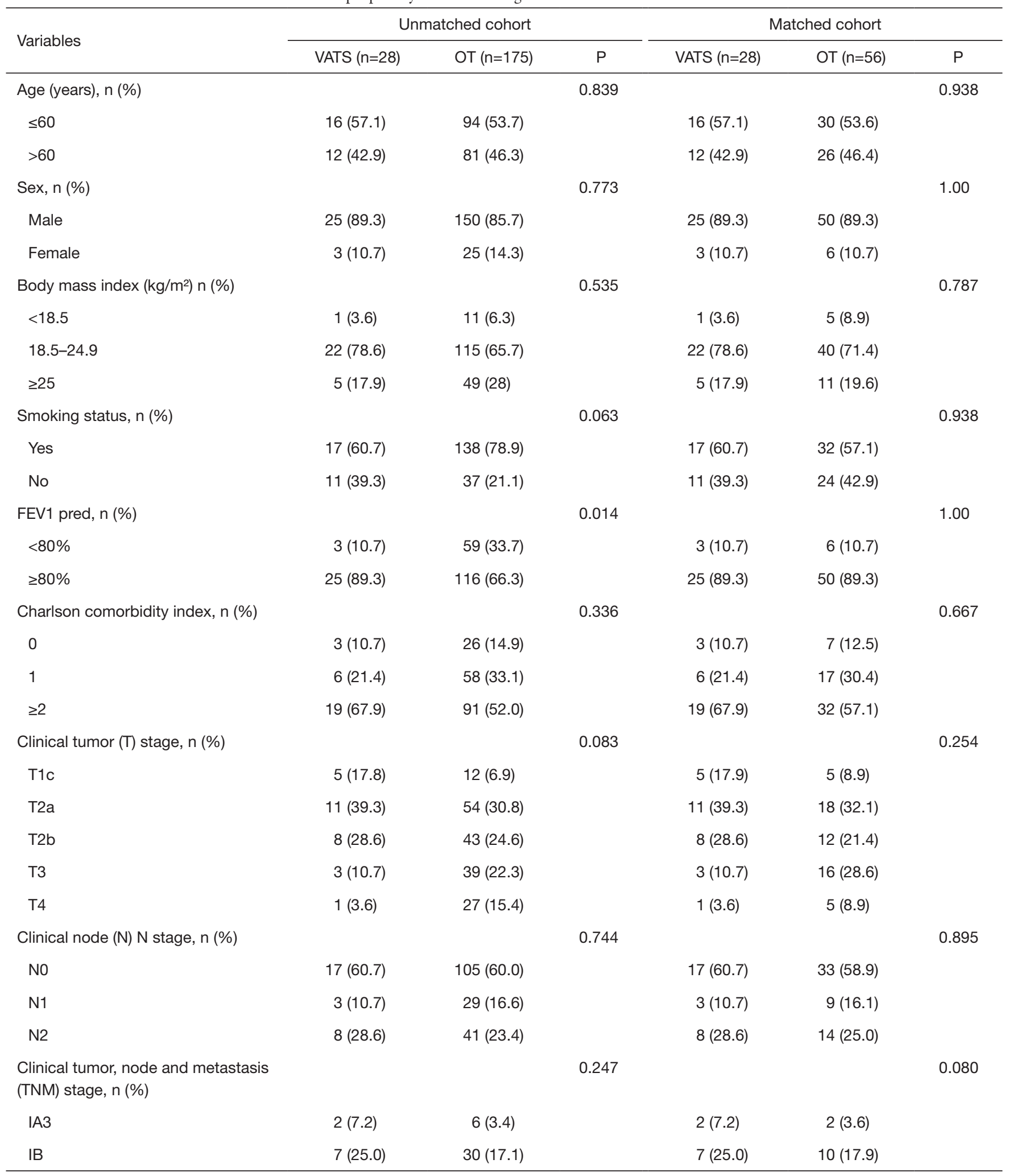

Table 1 (continued) 
Table 1 (continued)

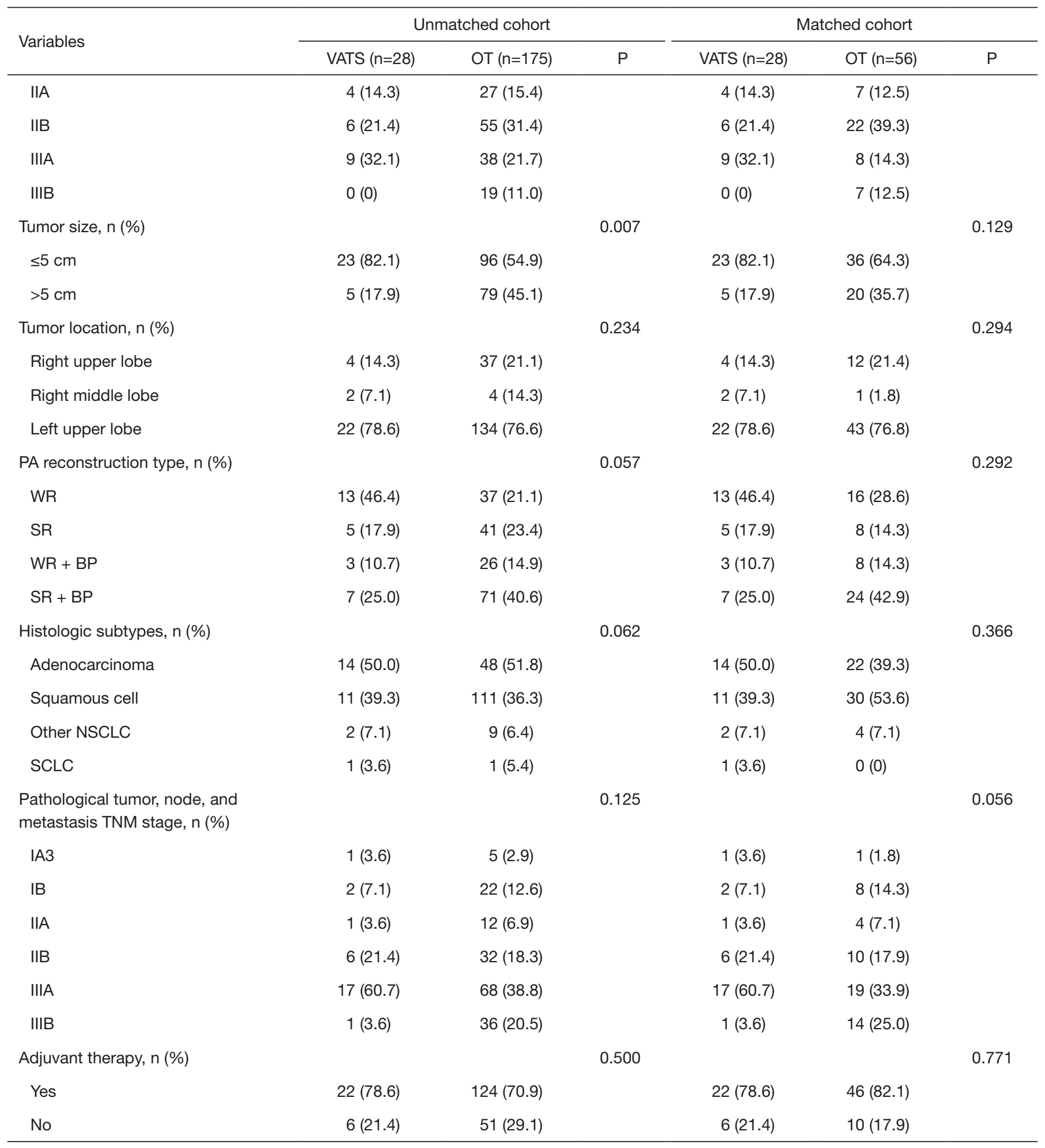

VATS, video-assisted thoracic surgery; OT, open thoracotomy; FEV1, forced expiratory volume in 1 second; PA, pulmonary artery; WR, wedge reconstruction; SR, sleeve reconstruction; BP, bronchial plasty; NSCLC, non-small cell lung cancer, SCLC, small cell lung cancer. 
Table 2 Surgical and postoperative outcomes before and after propensity score matching

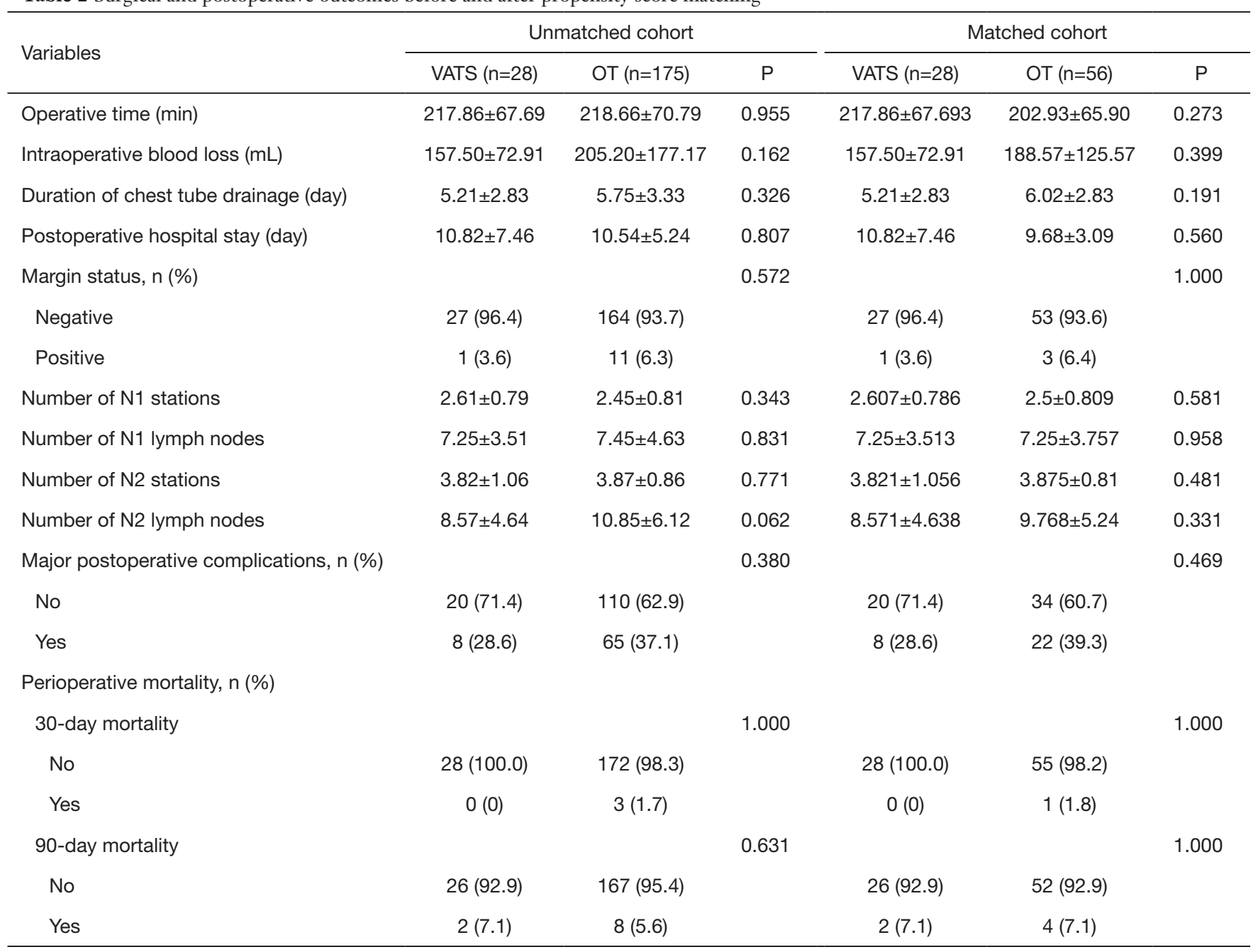

VATS, video-assisted thoracic surgery; OT, open thoracotomy

There were no differences between the two groups in postoperative morbidity or mortality both before and after matching.

\section{Survival outcomes}

The median follow-up period was 43 (range, 6 to 158) months and 44 (range, 7 to 158 ) months in the overall cohort and matched cohort, respectively. The 5-year OS and RFS of the overall cohort were $47.9 \%$ and $42.1 \%$, respectively. A log-rank test showed that there were no differences $(\mathrm{P}>0.05)$ between the OT and VATS groups in OS and RFS. The 5-year OS was comparable between the VATS and OT groups both in the overall cohort [49.9\% (95\% CI: $32.8-76.0 \%$ ) vs. $47.6 \%$ (95\% CI: 40.2-56.2\%), respectively] and matched cohort
[49.9\% (95\% CI: $32.8-76.0 \%)$ vs. $58.1 \%$ (95\% CI: $45.4-$ $74.3 \%$ ), respectively] (Figure 4A,4B). The 5-year RFS was also comparable between the VATS and OT groups both in the overall cohort [39.1\% (95\% CI: 23.4-65.2\%) vs. 42.6\% (95\% CI: 35.4-51.1\%), respectively] and matched cohort [39.1\% (95\% CI: $23.4-65.2 \%$ ) vs. $44.1 \%$ (95\% CI: $32.1-60.5 \%$ ), respectively] (Figure 4C,4D). The results of univariable and multivariable analyses for OS and RFS are shown in Table 3. Number of dissected N2 lymph nodes $\geq 11$ (hazard ratio, 0.36; 95\% CI: 0.14 to $0.93, \mathrm{P}=0.035$ ) was the only favorable factor for OS. Charlson comorbidity index $\geq 2$ (hazard ratio, 0.43 ; 95\% CI: 0.19 to $0.97, \mathrm{P}=0.041$ ) and number of dissected N2 lymph nodes $\geq 11$ (hazard ratio, 0.22 ; $95 \%$ CI: 0.16 to 0.85 , $\mathrm{P}=0.019)$ were both favorable factors for RFS. Of importance, the VATS procedure was not found to be an important 


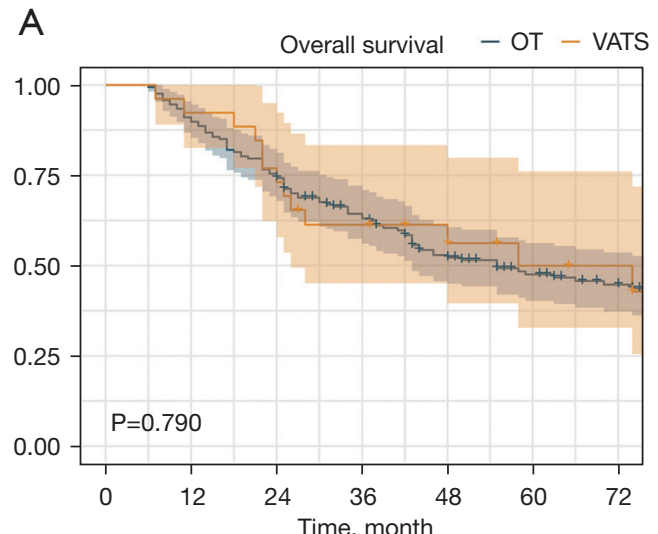

C
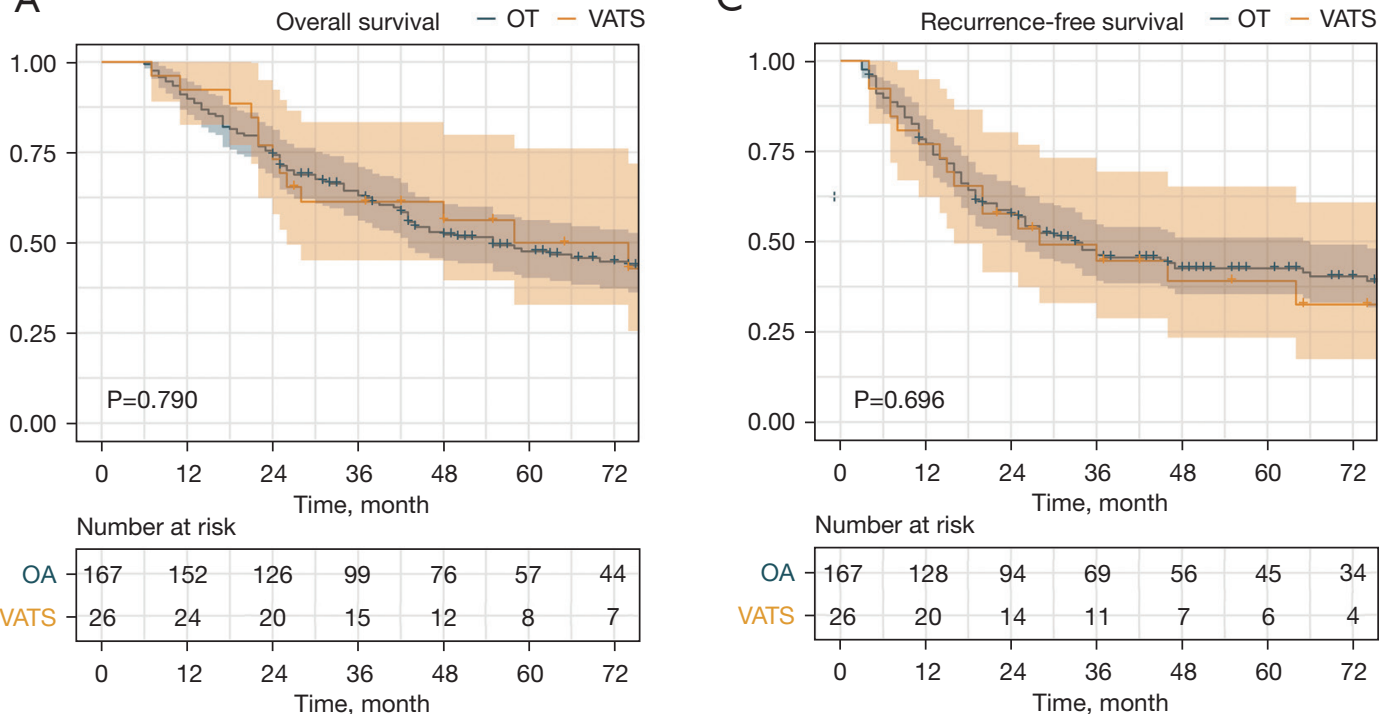

B

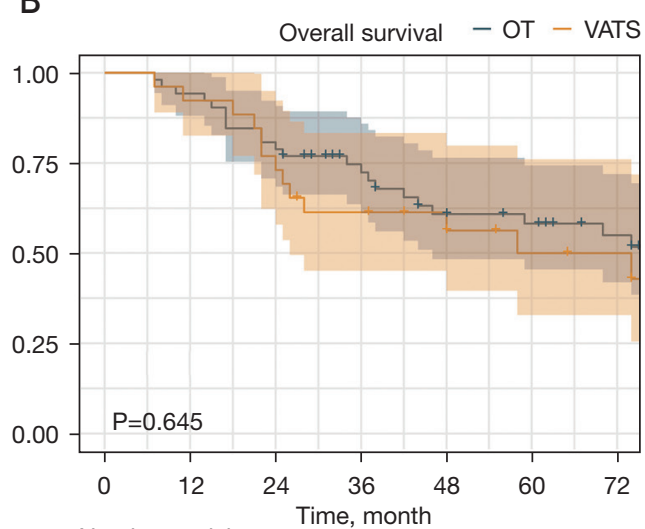

D
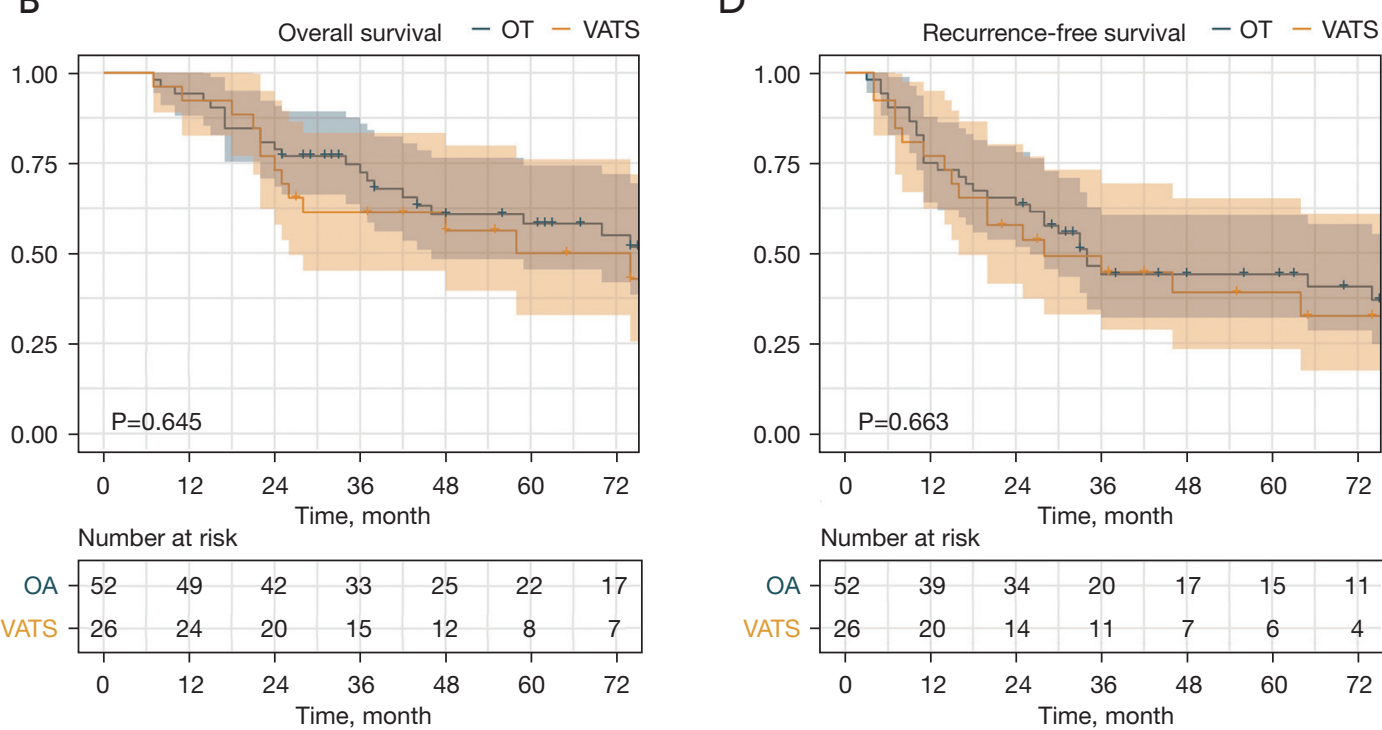

Figure 4 Kaplan-Meier survival estimates for patients who underwent PAA by VATS and open thoracotomy. (A,B) OS of patients in the overall cohort and matched cohort, respectively; (C,D) RFS of patients in the overall cohort and matched cohort, respectively. PAA, pulmonary artery angioplasty; OT, open thoracotomy; VATS, video-assisted thoracic surgery; OS, overall survival; RFS, recurrence-free survival.

prognostic factor of OS (hazard ratio, 1.17; $95 \%$ CI: 0.60 to 2.30, $\mathrm{P}=0.647$ ) or RFS (hazard ratio, 1.14 ; $95 \% \mathrm{CI}$ : 0.62 to $2.10, \mathrm{P}=0.666)$.

\section{Discussion}

In the present study, we evaluated the short- and longterm outcomes of patients who underwent VATS versus open PAA for lung cancer based on the WCLC database. To the best of our knowledge, this is the largest case series of PAA for lung cancer surgery with 203 cases included. In our study, the postoperative morbidity was $35.96 \%$ (73/203) and the postoperative mortality within 30 and 90 days were $1.5 \%(3 / 203)$ and $4.9 \%(10 / 203)$, respectively. The 5 -year OS was $47.9 \%$. Both the short- and long-term outcomes of our cohort were not inferior to those reported in previous 
Table 3 Univariable and multivariable cox regression for overall survival and recurrence-free survival after propensity score matching

\begin{tabular}{|c|c|c|c|c|c|c|c|c|c|c|c|c|}
\hline \multirow{2}{*}{ Variables } & \multicolumn{6}{|c|}{ OS } & \multicolumn{6}{|c|}{ RFS } \\
\hline & $\mathrm{HR}$ & $95 \% \mathrm{Cl}$ & $\mathrm{P}$ & $\mathrm{HR}$ & $95 \% \mathrm{Cl}$ & $\mathrm{P}$ & $\mathrm{HR}$ & $95 \% \mathrm{Cl}$ & $\mathrm{P}$ & $\mathrm{HR}$ & $95 \% \mathrm{Cl}$ & $\mathrm{P}$ \\
\hline \multicolumn{13}{|l|}{ Age } \\
\hline \multicolumn{13}{|l|}{$\leq 60$ years (ref) } \\
\hline \multicolumn{13}{|l|}{ Sex } \\
\hline \multicolumn{13}{|l|}{ Male (ref) } \\
\hline Female & 1.28 & $0.45-3.64$ & 0.642 & & & & 1.35 & $0.57-3.21$ & 0.491 & & & \\
\hline \multicolumn{13}{|c|}{ Body mass index (kg/m²) } \\
\hline$\geq 25$ & 1.77 & $0.22-14.19$ & 0.591 & & & & 0.72 & $0.20-2.59$ & 0.616 & & & \\
\hline \multicolumn{13}{|l|}{ Smoking status } \\
\hline \multicolumn{13}{|l|}{ Yes (ref) } \\
\hline No & 1.06 & $0.54-2.08$ & 0.875 & & & & 1.11 & $0.61-2.02$ & 0.732 & & & \\
\hline \multicolumn{13}{|l|}{ FEV1 pre } \\
\hline \multicolumn{13}{|l|}{$<80 \%$ (ref) } \\
\hline$\geq 80 \%$ & 2.13 & $0.51-8.87$ & 0.300 & & & & 1.79 & $0.56-5.78$ & 0.329 & & & \\
\hline \multicolumn{13}{|c|}{ Charlson comorbidity index } \\
\hline Right middle lobe & 1.22 & $0.24-6.07$ & 0.810 & & & & 0.83 & $0.18-3.85$ & 0.811 & & & \\
\hline Left upper lobe & 0.98 & $0.41-2.35$ & 0.956 & & & & 0.69 & $0.33-1.44$ & 0.328 & & & \\
\hline \multicolumn{13}{|l|}{ Approach } \\
\hline \multicolumn{13}{|l|}{ OT (ref) } \\
\hline VATS & 1.17 & $0.60-2.30$ & 0.647 & & & & 1.14 & $0.62-2.10$ & 0.666 & & & \\
\hline \multicolumn{13}{|l|}{ N1 number } \\
\hline \multicolumn{13}{|l|}{$\leq 5$ (ref) } \\
\hline $6-10$ & 1.38 & $0.61-3.11$ & 0.444 & & & & 1.25 & $0.63-2.47$ & 0.528 & & & \\
\hline$\geq 11$ & 1.64 & $0.61-4.38$ & 0.324 & & & & 1.24 & $0.51-3.05$ & 0.633 & & & \\
\hline
\end{tabular}

Table 3 (continued) 
Table 3 (continued)

\begin{tabular}{|c|c|c|c|c|c|c|c|c|c|c|c|c|}
\hline \multirow{2}{*}{ Variables } & \multicolumn{6}{|c|}{ OS } & \multicolumn{6}{|c|}{ RFS } \\
\hline & HR & $95 \% \mathrm{Cl}$ & $\mathrm{P}$ & $\mathrm{HR}$ & $95 \% \mathrm{Cl}$ & $\mathrm{P}$ & $\mathrm{HR}$ & $95 \% \mathrm{Cl}$ & $\mathrm{P}$ & $\mathrm{HR}$ & $95 \% \mathrm{Cl}$ & $\mathrm{P}$ \\
\hline \multicolumn{13}{|l|}{ N2 number } \\
\hline \multicolumn{13}{|l|}{$\leq 5$ (ref) } \\
\hline$\geq 11$ & 0.36 & $0.14-0.93$ & 0.035 & 0.36 & $0.14-0.93$ & 0.035 & 0.37 & $0.16-0.86$ & 0.020 & 0.22 & $0.16-0.85$ & 0.019 \\
\hline \multicolumn{13}{|c|}{ Type of PA reconstruction } \\
\hline \multicolumn{13}{|l|}{ WR (ref) } \\
\hline SR & 1.31 & $0.50-3.39$ & 0.583 & & & & 1.61 & $0.71-3.67$ & 0.255 & & & \\
\hline \multicolumn{13}{|c|}{ Histology subtypes } \\
\hline \multicolumn{13}{|c|}{ Adenocarcinoma (ref) } \\
\hline Squamous cell & 0.79 & $0.40-1.57$ & 0.505 & & & & 1.16 & $0.63-2.15$ & 0.640 & & & \\
\hline Other NSCLC & 0.89 & $0.26-3.03$ & 0.847 & & & & 0.54 & $0.12-2.32$ & 0.403 & & & \\
\hline SCLC & 0.30 & $0.04-2.23$ & 0.238 & & & & 0.88 & $0.26-2.99$ & 0.836 & & & \\
\hline \multicolumn{13}{|l|}{ pTNM } \\
\hline \multicolumn{13}{|l|}{ I (ref) } \\
\hline II & 0.48 & $0.17-1.36$ & 0.167 & & & & 0.77 & $0.30-2.01$ & 0.773 & & & \\
\hline
\end{tabular}

*, multivariable cox regression analysis for OS was not conducted because only one factor reached the P<0.1 threshold. OS, overall survival; DFS, disease-free survival; VATS, video-assisted thoracic surgery; OT, open thoracotomy; NSCLC, non-small cell lung cancer; $\mathrm{HR}$, hazard ratio; $\mathrm{Cl}$, confidence interval; WR, wedge reconstruction; SR, sleeve reconstruction; BP, bronchial plasty; FEV1 pre, forced expiratory volume in 1 second percent predicted.

studies, which reported a postoperative morbidity of 23.0 $52.2 \%$, a postoperative mortality of $0-5.4 \%$, and a 5 -year OS of 39.4-50.2\% (5,6,23-25).

Lobectomy with PAA performed using VATS is much more difficult than that performed by OT. Previous studies of VATS PAA were focused on surgical techniques and procedures using different approaches, perioperative management, and outcomes $(13,15,16,26-29)$. There is still a lack of evaluation of the short- and long-term outcomes of complete VATS PAA compared with an open approach.
Therefore, we conducted the first comparative study to evaluate the short- and long-term outcomes of VATS versus OT PAA for lung cancer. Given the presence of a potential learning curve, cases included in the VATS group might be easier cases, and there might be selection bias. In the unmatched cohort, there were less cases with tumor $>5 \mathrm{~cm}$ in the VATS group. To reduce the impact of baseline characteristic differences and obtain convincing statistically significant results, PSM analysis was adopted to balance all known confounders between the VATS and OT groups. 
After matching, the bias from known confounders was minimized.

To the best of our knowledge, previous studies rarely reported on the conversion rate of VATS PAA. In this cohort, the total conversion rate of VATS PAA was 25\% (7/28). The reasons included one cases of severe lobar hilum invasion, one case of chest wall invasion, and one case of superior vena cava invasion. Therefore, careful evaluation of the extent of tumor invasion was crucial before selecting VATS procedure for a lung resection that needed PAA. Surgeons also need to prepare for conversion to OT as an alternative. The operative time in the VATS group was longer, probably due to more complex operative techniques using thoracoscopy. Using thoracoscopy, sufficient access to the main PA and control of the proximal and distal PA are much more difficult $(13,15,16,21)$. In addition, suturing using thoracoscopy was also more complicated due to relatively limited space and operative angle $(18,19)$. The VATS procedure was associated with less intraoperative blood loss. The incidences of positive margins were $3.6 \%(1 / 28)$ in the VATS group and $6.3 \%$ $(11 / 175)$ in the OT group, respectively. The number of lymph nodes dissected were similar in the two groups. This indicated that the VATS procedure was as effective as the OT procedure in achieving a radical resection during lung surgery complicated with PAA. The incidence of major complications was numerically lower in the VATS group before and after matching. Although there was no postoperative death in 30 days in the VATS group, while three patients $(1.7 \%)$ died within 30 days following surgery in the OT group, the statistic test was not significant $(\mathrm{P}=0.631)$. The most lethal complication after $\mathrm{PAA}$ was rupture of the reconstructed vascular wall. One patient in the OT group, who underwent double sleeve reconstruction, suffered massive bleeding due to rupture of the pulmonary artery and died, while there were no patients in the VATS group, who experienced such catastrophic outcomes. Taken together, although the differences between the two groups were not significant, the lower postoperative morbidity and mortality suggest slight advantages of VATS PAA for lung cancer surgery.

Importantly, the VATS and OT procedures had equivalent oncological outcomes in patients with PAA. In both overall and matched cohorts, there were no significant differences in 5-year OS and RFS between the VATS and OT groups in this study. Furthermore, the multivariable analysis demonstrated that the surgical approach was not an important prognostic factor for 5-year OS and RFS, but only the number of dissected N2 lymph nodes $\geq 11$ was an independent favorable factor for long-term survival. Therefore, we carefully conclude that VATS PAA for lung cancer is equivalent to thoracotomy based on oncological outcomes. Independent predictors of RFS in this cohort also included Charlson comorbidity index. Interestingly, a Charlson comorbidity index $\geq 2$ was a favorable prognostic factor for RFS. This might be attributed to the small sample size of this cohort.

Our study has several limitations. First, the nature of this retrospective study from a single institution may have led to unmeasured confounders, and the results may be influenced by potential bias even though PSM analysis was performed. Second, VATS PAA is a very challenging technique, such that the number of cases included in the VATS group is small, and the outcomes may also be influenced by a learning curve. Moreover, generalizations of the obtained results to other clinical settings must be made cautiously, because the data presented for the VATS group were derived from surgeons who are extremely experienced with minimally invasive and open lung surgery. Given the above reasons, whether the findings translate into real-world practice needs further confirmation.

\section{Conclusions}

VATS PAA is associated with comparable short- and longterm outcomes in selected patients with lung cancer when compared with OT. Careful evaluation of the extent of tumor invasion was crucial before selecting VATS procedure for a lung resection that needed PAA. However, VATS lobectomy complicated with PAA is technically demanding, and each surgeon should perform operations within his/her own ability.

\section{Acknowledgments}

Funding: This study was supported by the Basic Science Program (2019YJ0077 to CL), Science and Technology Department of Sichuan Province, and the 1.3.5 Project for Disciplines of Excellence (ZYJC18009 to JM, and ZYGD18021 to LL), West China Hospital, Sichuan University.

\section{Footnote}

Reporting Checklist: The authors have completed the STROBE reporting checklist. Available at https://dx.doi. 
org/10.21037/tlcr-21-607

Data Sharing Statement: Available at https://dx.doi. org/10.21037/tlcr-21-607

Conflicts of Interest: All authors have completed the ICMJE uniform disclosure form (available at https://dx.doi. org/10.21037/tlcr-21-607). The authors have no conflicts of interest to declare.

Ethical Statement: The authors are accountable for all aspects of the work in ensuring that questions related to the accuracy or integrity of any part of the work are appropriately investigated and resolved. The protocol of this study was approved by the institutional review board of West China Hospital (No. 2020-939). All procedures performed in this study involving human participants were in accordance with the Declaration of Helsinki (as revised in 2013). Written informed consent was obtained from each patient before surgery.

Open Access Statement: This is an Open Access article distributed in accordance with the Creative Commons Attribution-NonCommercial-NoDerivs 4.0 International License (CC BY-NC-ND 4.0), which permits the noncommercial replication and distribution of the article with the strict proviso that no changes or edits are made and the original work is properly cited (including links to both the formal publication through the relevant DOI and the license). See: https://creativecommons.org/licenses/by-nc-nd/4.0/.

\section{References}

1. Deslauriers J, Tronc F, Grégoire J. History and current status of bronchoplastic surgery for lung cancer. Gen Thorac Cardiovasc Surg 2009;57:3-9.

2. Shrager JB, Lambright ES, McGrath CM, et al. Lobectomy with tangential pulmonary artery resection without regard to pulmonary function. Ann Thorac Surg 2000;70:234-9.

3. Rendina EA, Venuta F, De Giacomo T, et al. Sleeve resection and prosthetic reconstruction of the pulmonary artery for lung cancer. Ann Thorac Surg 1999;68:9951001; discussion 1001-2.

4. Cerfolio RJ, Bryant AS. Surgical techniques and results for partial or circumferential sleeve resection of the pulmonary artery for patients with non-small cell lung cancer. Ann Thorac Surg 2007;83:1971-6; discussion 1976-7.
5. Alifano M, Cusumano G, Strano S, et al. Lobectomy with pulmonary artery resection: morbidity, mortality, and long-term survival. J Thorac Cardiovasc Surg 2009;137:1400-5.

6. Venuta F, Ciccone AM, Anile M, et al. Reconstruction of the pulmonary artery for lung cancer: long-term results. J Thorac Cardiovasc Surg 2009;138:1185-91.

7. Park JS, Yang HC, Kim HK, et al. Sleeve lobectomy as an alternative procedure to pneumonectomy for non-small cell lung cancer. J Thorac Oncol 2010;5:517-20.

8. Ma Z, Dong A, Fan J, et al. Does sleeve lobectomy concomitant with or without pulmonary artery reconstruction (double sleeve) have favorable results for non-small cell lung cancer compared with pneumonectomy? A meta-analysis. Eur J Cardiothorac Surg 2007;32:20-8.

9. Vannucci J, Matricardi A, Potenza R, et al. Lobectomy with angioplasty: which is the best technique for pulmonary artery reconstruction? J Thorac Dis 2018;10:S1892-8.

10. Maurizi G, D'Andrilli A, Venuta F, et al. Reconstruction of the bronchus and pulmonary artery. J Thorac Dis 2016;8:S168-80.

11. Pan X, Fu S, Shi J, et al. The early and long-term outcomes of completion pneumonectomy: report of 56 cases. Interact Cardiovasc Thorac Surg 2014;19:436-40.

12. Santambrogio L, Cioffi U, De Simone M, et al. Videoassisted sleeve lobectomy for mucoepidermoid carcinoma of the left lower lobar bronchus: a case report. Chest 2002;121:635-6.

13. Nakanishi R, Yamashita T, Oka S. Initial experience of video-assisted thoracic surgery lobectomy with partial removal of the pulmonary artery. Interact Cardiovasc Thorac Surg 2008;7:996-1000.

14. Nakanishi R, Oka S, Odate S. Video-assisted thoracic surgery major pulmonary resection requiring control of the main pulmonary artery. Interact Cardiovasc Thorac Surg 2009;9:618-22.

15. Kamiyoshihara $M$, Nagashima T, Ibe T, et al. A tip for controlling the main pulmonary artery during videoassisted thoracic major pulmonary resection: the outsidefield vascular clamping technique. Interact Cardiovasc Thorac Surg 2010;11:693-5.

16. Zhang $Z$, Huang J, Yin R, et al. A new technique for partial removal of the pulmonary artery in video-assisted thoracic surgical lobectomy. J Thorac Cardiovasc Surg 2012;144:512-4.

17. Han Y, Zhou S, Yu D, et al. Video-assisted thoracic surgery (VATS) left upper sleeve lobectomy with partial pulmonary 
artery resection. J Thorac Dis 2013;5 Suppl 3:S301-3.

18. Huang J, Li J, Qiu Y, et al. Thoracoscopic double sleeve lobectomy in 13 patients: a series report from multicenters. J Thorac Dis 2015;7:834-42.

19. Liu L, Mei J, Pu Q, et al. Thoracoscopic bronchovascular double sleeve lobectomy for non-small-cell lung cancer. Eur J Cardiothorac Surg 2014;46:493-5.

20. Vandenbroucke JP, von Elm E, Altman DG, et al. Strengthening the Reporting of Observational Studies in Epidemiology (STROBE): explanation and elaboration. PLoS Med 2007;4:e297.

21. Liu C, Ma L, Pu Q, et al. Troubleshooting complicated hilar anatomy via prophylactically clamping the pulmonary artery: three videos demonstrating three techniques. Ann Transl Med 2018;6:365.

22. Xu X, Huang J, Yin W, et al. Pulmonary arterioplasty using video-assisted thoracic surgery mechanical suture technique. J Thorac Dis 2016;8:617-27.

23. Ma Q, Liu D, Guo Y, et al. Surgical techniques and results of the pulmonary artery reconstruction for patients with central non-small cell lung cancer. J Cardiothorac Surg 2013;8:219.

24. Galetta D, Borri A, Gasparri R, et al. Surgical

Cite this article as: Liu C, Yang Z, Guo C, Zhu Y, Pu Q, Mei J, Ma L, Lin F, Liu L. Lobectomy with pulmonary artery angioplasty for lung cancer using video-assisted thoracic surgery versus open thoracotomy: a retrospective propensity matched analysis. Transl Lung Cancer Res 2021;10(10):39433956. doi: $10.21037 /$ tlcr-21-607
Techniques and Long-Term Results of Pulmonary Artery Reconstruction in Patients With Lung Cancer. Ann Thorac Surg 2015;100:1196-202; discussion 1202.

25. Kojima F, Yamamoto K, Matsuoka K, et al. Factors affecting survival after lobectomy with pulmonary artery resection for primary lung cancer. Eur J Cardiothorac Surg 2011;40:e13-20.

26. Xu K, Zhang Z, Zhao J, et al. Partial removal of the pulmonary artery in video-assisted thoracic surgery for non-small cell lung cancer. J Biomed Res 2013;27:310-7.

27. Gonzalez-Rivas D, Delgado M, Fieira E, et al. Single-port video-assisted thoracoscopic lobectomy with pulmonary artery reconstruction. Interact Cardiovasc Thorac Surg 2013;17:889-91.

28. Dong YN, Sun N, Ren Y, et al. Thoracoscopic left upper lobectomy with systematic lymph nodes dissection under left pulmonary artery clamping. J Thorac Dis 2014;6:1855-60.

29. Jiang L, Wu L, Roque SR, et al. A novel tourniquet technique for transient pulmonary artery occlusion during video-assisted thoracoscopic surgery. J Thorac Cardiovasc Surg 2018;156:816-8.

(English Language Editor: B. Meiser) 